\title{
Hairy polyp of the rhinopharynx - a rare and unrecognized entity
}

\author{
Dan Cristian GHEORGHE ${ }^{1,5}$, Adina ZAMFIR-CHIRU-ANTON², LE ENE $^{3}$, AE STANCIU ${ }^{4,5}$ \\ ${ }^{1}$ ENT Department, "MS Curie" Hospital, Bucharest, Romania \\ 2ENT Department, "Gr. Alexandrescu" Hospital, Bucharest, Romania \\ ${ }^{3}$ ENT Department, City Hospital, Slatina, Romania \\ ${ }^{4}$ Department of Carcinogenesis and Molecular Biology, Institute of Oncology, Bucharest, Romania \\ 5"Carol Davila" University of Medicine and Pharmacy, Bucharest, Romania
}

\begin{abstract}
Hairy polyps are tumors that have been described in the literature as congenital masses usually found in the nasopharynx. Clinical symptoms are a consequence of disease dimensions and anatomical positioning. Although rare, this kind of tumors can be mistaken for neurologic impairment of an infant. Histological examination is the base for classification of this malformation. Earlier management of the disease could prevent possible complications (of the airway) and allow a better long-term growth of the patient.
\end{abstract}

Keywords: hairy polyps, rhinopharynx, tumor

\section{INTRODUCTION}

Hairy polyps are tumors that have been described in the literature as congenital masses usually found in the nasopharynx [1]. Their incidence is low $[2,3]$. Histologically, they contain both ectodermal and mesodermal tissues [2]. The point of insertion into the pharynx can vary: most of the tumors are linked to the soft palate [2], but some authors published palatopharyngeus muscle as the origin point [4].

Clinical symptoms are a consequence of disease dimensions and anatomical positioning. Disordered breathing and dysphagia can be found [5]. Diagnosis can be suspected at clinical exmination of the patient and completing the evaluation with imagistics: computed tomography scanning (CT) or magnetic resonance imaging (MRI). Surgical removal is the therapeutic approach and pathologic examination confirms the diagnosis.
There is no clear origin of the tumor but, most of the authors hypothesize the branchial origin of this malformation [6]. Some even presumed these tumors to be the equivalent of accesory auricles trapped into the nasopharynx [7]. In this hypothesis, some authors consider hairy polyps origins to be located in the lower third of the Eustachian tube [8].

\section{CASE PRESENTATION}

We present a case of a 1-year-old girl, referred to our department for feeding problems, with intermittent choking, lasting from birth but worsened during the last month. At clinical examination we noted a tumor originating from the posterior face of the soft palate. A computed tomography (CT) scanning showed the tumor advancing into the proximal esophagus for at least $3 \mathrm{~cm}$ (figures 1-2). 

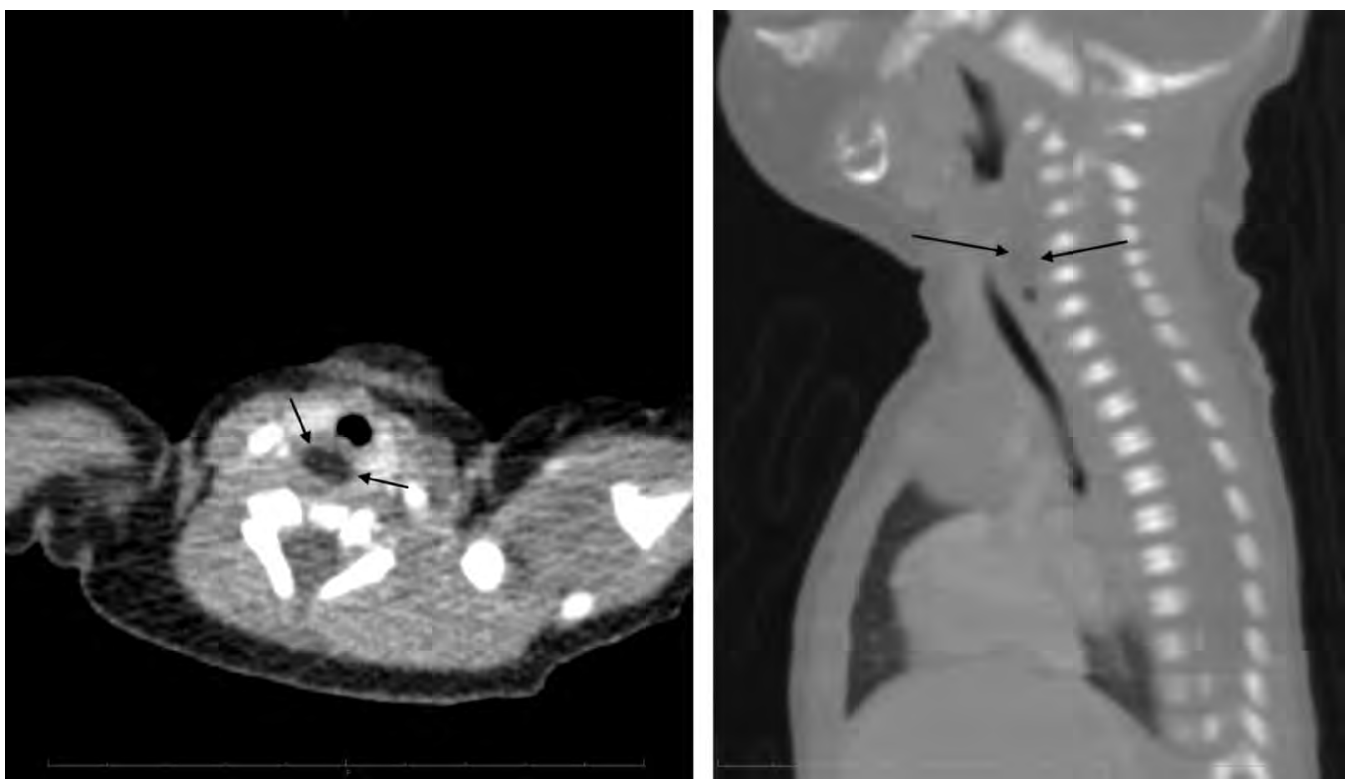

FIGURES 1-2. CT scanning of the presented patient, in axial (1) and sagittal axis. Black arrows demonstrate the contour of the tumor progressing inferiorly into the esophagus

Under general anesthesia (GA), we found a pedunculated tumor, appearantly covered with skin and originating from the posterior left face of the soft palate. It could be surgically removed and its origin cauterized (figure 3 ) after soft palate retraction, under direct vision. Histology showed keratinizing squamous epithelium covering fat tissue and cartilage. No complications were noted postoperatively. The child recovered quickly and no similar symptoms could be elicited at 1-month follow-up.

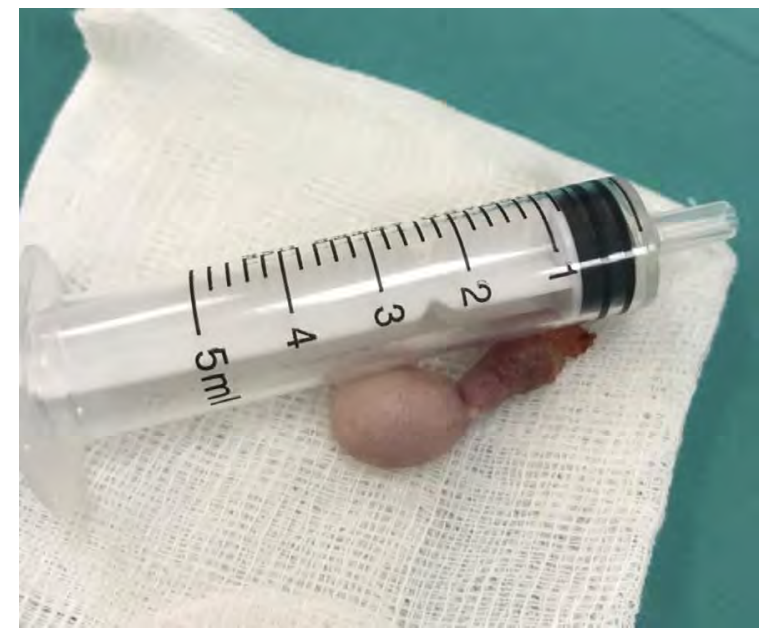

FIGURE 3. Extracted tumor

\section{DISCUSSION}

Although rare, this kind of tumors can be mistaken for neurologic impairment of an infant. Its congenital nature does not bring much light into the differential diagnosis, regarding deglutition troubles after birth.

Good clinical examination in a child can be difficult due to high position of the hyoid-base of tongue complex or the presence of an endotracheal tube [3]. Detailed examination can sometimes need nasopharyngeal fibroscopy and to a much lesser extent general anesthesia.

Tumors noted in the naso-pharyngeal region are usually benign or congenital. Teratomas, foreign bodies, glioma, meningo-encephalocele, lingual thyroid gland, vallecular cysts must all be differentiated from hairy polyps. Histological examination is the base for classification of this malformation.

Also, pedicle insertion point could bring clues to its origin. Our case demonstrated a long insertion pedicle into the left rhinopharynx.

Theoretically, these esophageal polyps, given the long pedicle, could eventually become dislodged from their esophageal position and threaten the airway at the level of the larynx $[9,10]$. We did not get a history of such symptoms, but only feeding difficulties as the only complaint.

\section{CONCLUSIONS}

Although rare, this malformation should be reminded whenever it is present from birth and has a typical appearance (hairy), especially if located posterior to the soft palate. Treatment is by surgical excision. Prognosis is good, with almost no recurrences reported. We think that earlier management of the disease could prevent possible complications (of the airway) and allow a better long-term growth of the patient. 


\section{REFERENCES}

1. Kalcioglu MT, Can C, Aydin NE. Unusual case of soft palate hairy polyp causing airway obstruction and review of the literature. J Pediatr Surg, 2010;45(12):e5-8.

2. Yilmaz $M$ et al. Congenital hairy polyp of the soft palate. Int J Pediatr

Otorhinolaryngol. 2012;76(1):5-8.

3. Budenz CL, Lesperance MM, Gebarski S. Hairy polyp of the pharynx obscured on physical examination by endotracheal tube, but diagnosed on brain imaging. Pediatr Radiol. 2005;35(11):1107-9.

4. Richter A et al. Congenital hairy polyp of the oropharynx presenting as an esophageal

Conflict of interest: none declared Financial support: none declared mass in a neonate, a case report and literature review. Int J Pediatr Otorhinolaryngol. 2016;80:26-9.

5. Koike $Y$ et al. Hairy polyp can be lethal even when small in size. Pediatr Int. 2013; 55(3):373-6.

6. Burns BV, Axon PR, Pahade A. 'Hairy polyp' of the pharynx in association with an ipsilateral branchial sinus: Evidence that the 'hairy polyp' is a second branchial arch malformation. J Laryngol Otol. 2001; 115(2):145-8.

7. Schuring AG. Accessory Auricle in the Nasopharynx. Laryngoscope, 1964;74:111-4.
8. Walker P. Dilated Eustachian tube orifice after endoscopic removal of hairy polyp. Otolaryngol Head Neck Surg. 2008; 139(1):162-3.

9. Cochet $B$ et al. Asphyxia caused by laryngeal impaction of an esophageal polyp. Arch Otolaryngol. 1980; 106(3):176-8.

10. Jarvis SJ, Bull PD. Hairy polyps of the nasopharynx. J Laryngol Otol. 2002; 116(6):467-9. 